\title{
OVARIAN CANCER CARE AT A HOSPITAL WITH AN IGCS GLOBAL CURRICULUM FELLOWSHIP
}

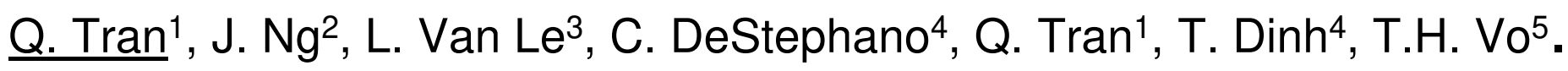 \\ ${ }^{1}$ Da Nang Oncology Hospital, Gynecologic Oncology, Da Nang, Vietnam. \\ ${ }^{2}$ National University Hospital, Obstetrics and Gynecology, Singapore, Singapore. \\ ${ }^{3}$ University of North Carolina School of Medicine, Gynecologic Oncology, Chapel Hill, USA. \\ ${ }^{4}$ Mayo Clinic Florida, Medical and Surgical Gynecology, Jacksonville, USA. \\ ${ }^{5} \mathrm{Da}$ Nang University of Medical Technology and Pharmacy, Pharmacy, Da Nang, Vietnam.
}

\section{Background and Aims:}

Worldwide more than half of all cancers are diagnosed in low- and middle-income countries (LMICs). In Vietnam, the incidence of cancer has tripled in the past 30 years, with 164, 671 cases diagnosed and 114, 871 deaths in 2018. Da Nang city, with a population of 1 million, is no exception to this trend. Despite the local availability of imaging and CA 125 testing, patients with adnexal masses are still operated on primarily by surgeons not trained in oncologic surgery. With the aim of mentoring early career professionals in gynecologic oncology in lower resource regions and equipping them to set up multidisciplinary teams and regional gynecologic cancer centers, the IGCS has been collaborating with the Da Nang Oncology Hospital (DOH) through the Global Curriculum \& Mentorship Program. This study determines the impact of the implementation of the IGCS global curriculum in July 2017 on ovarian cancer care in Da Nang, Vietnam.

\section{Methods:}

We performed a retrospective review of consecutive ovarian cancer cases treated since the start of the IGCS Global Curriculum fellowship in July, 2017 to March, 2019 at DOH. There were 2 groups of patients: those who underwent surgery for ovarian cancer at a general hospital then referred to $\mathrm{DOH}$ and those who were diagnosed and treated primarily at $\mathrm{DOH}$. Clinical parameters of the 2 groups were obtained and Chi-Squared analysis was used to compare the 2 groups.

\section{Results}

Between July, 2017 and March, 2019, 65 consecutive ovarian cancer cases were treated at $\mathrm{DOH} .27$ patients were initially treated at a general hospital and referred to $\mathrm{DOH}$, and 38 patients were diagnosed and treated at $\mathrm{DOH}$. Correct diagnosis was rendered in $55 \%$ of referred patients compared to $97 \%$ of patients diagnosed initially at $\mathrm{DOH}(\mathrm{p}=.000003)$
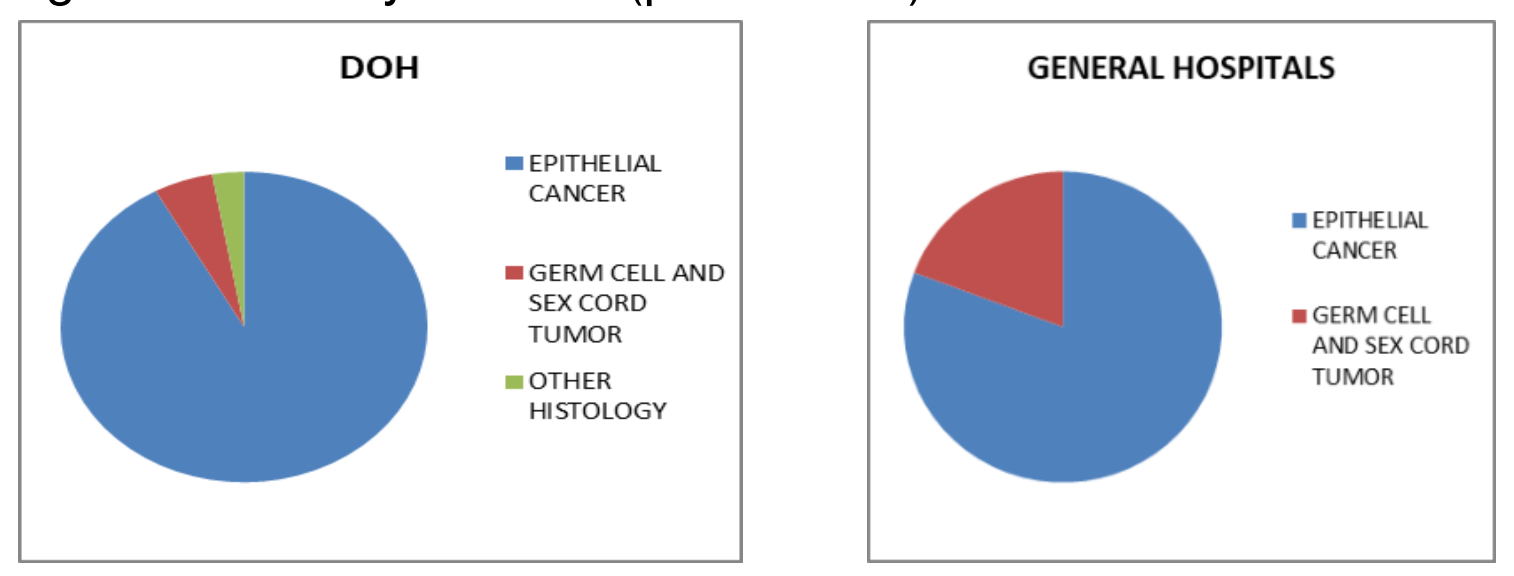

Figure 1: Histology of Cancer types diagnosed at $\mathrm{DOH}$ and

\section{General Hospital.}

$4(14 \%)$ of patients received adequate staging surgery at an outside hospital versus $(89 \%)$ of patients at $\mathrm{DOH}(\mathrm{p}<.000001)$. Patients were also more likely to have optimal surgery, defined as no gross residual disease after initial surgery if they were treated initially at $\mathrm{DOH}$ (45\%v $30 \%, \mathrm{p}=0.0005)$. Patients treated initially at a general hospital were more likely to require a second surgery within 6 months of initial diagnosis $(70 \%$ vs $42 \%, p=.0009)$.
Initial surgery were more likely to have been done by laparoscopy at $\mathrm{DOH}$ than at a general hospital (46\% vs $7 \%, \mathrm{p}<.000001)$. All of these patients diagnosed with laparoscopy at $\mathrm{DOH}$ were treated with neoadjuvant chemotherapy and $16(43 \%)$ went on to have interval cytoreduction. The optimal cytoreduction rate in this group was $40 \%$.

$9(34 \%)$ of patients who had attempted cytoreduction at the outside hospital (all via laparotomy) went on to have neoadjuvant chemotherapy. $30 \%$ of these patients were able to have a second interval cytoreduction surgery and $30 \%$ had optimal cytoreduction. All of the interval cytoreduction surgery in this group of patients were done at $\mathrm{DOH}$.

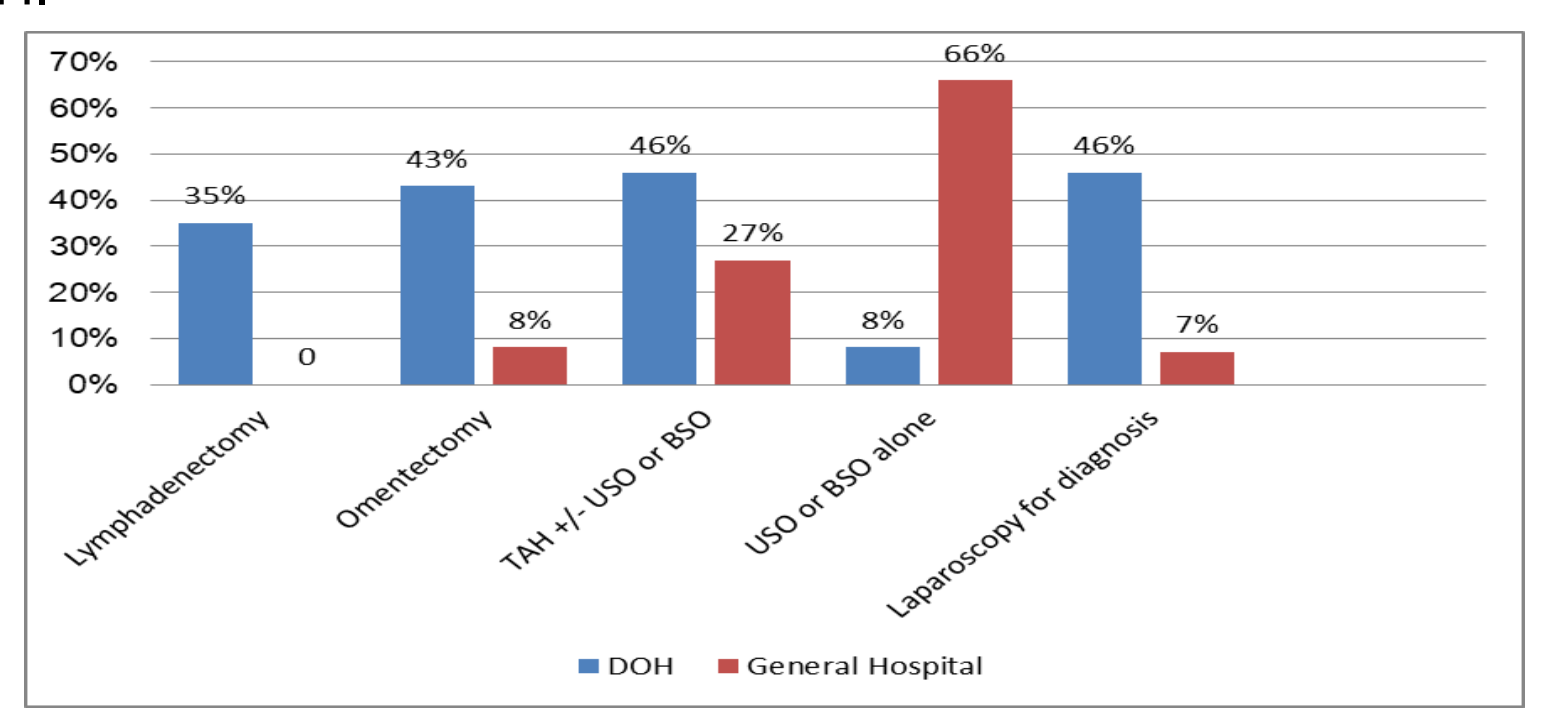

Figure 2: Procedures done by hospital at initial diagnosis (percent of cases at presentation)

*There were no lymphadenectomy done at primary surgery at the general hospitals

Referral to
DOH

Figure 3: Treatment at $\mathrm{DOH}$ by site of initial surgery for ovarian cancer Time to chemotherapy did not differ between the 2 groups. Germ cell tumors were more likely to be diagnosed at a general hospital than at $\mathrm{DOH}(\mathrm{p}=.04)$.

\section{Conclusions:}

In Da Nang, receiving initial care for ovarian cancer at a hospital affiliated with the IGCS Global Curriculum Fellowship more often resulted in correct diagnosis and appropriate therapy. After only 2 years, it is too early to determine whether this translates to an improvement in survival for patients with ovarian cancer in the area. However, indicators such as rate of laparoscopy versus laparotomy for diagnosis, correct initial diagnosis, adequate surgical staging favor patients who were treated at a hospital associated with the IGCS Fellowship curriculum. There is a need to educate Vietnamese physicians about the importance of timely referrals of abdomino-pelvic masses suspicious for ovarian cancer to an oncology hospital. This is especially important for younger patients in whom a germ cell ovarian tumor is more likely. 